\title{
Regional snow-avalanche detection using object-based image analysis of near-infrared aerial imagery
}

\author{
Karolina Korzeniowska ${ }^{1,2}$, Yves Bühler ${ }^{3}$, Mauro Marty ${ }^{4}$, and Oliver Korup ${ }^{2}$ \\ 13D Mapping, BSF Swissphoto GmbH, Schönefeld, 12529, Germany \\ ${ }^{2}$ Geohazards Research Group, University of Potsdam, Potsdam, 14476, Germany \\ ${ }^{3}$ WSL Institute for Snow and Avalanche Research SLF, Davos, 7260, Switzerland \\ ${ }^{4}$ Swiss Federal Institute for Forest, Snow and Landscape Research WSL, Birmensdorf, 8903, Switzerland \\ Correspondence to: Karolina Korzeniowska (karolina.korzeniowska@bsf-swissphoto.com)
}

Received: 30 March 2017 - Discussion started: 10 April 2017

Accepted: 24 August 2017 - Published: 23 October 2017

\begin{abstract}
Snow avalanches are destructive mass movements in mountain regions that continue to claim lives and cause infrastructural damage and traffic detours. Given that avalanches often occur in remote and poorly accessible steep terrain, their detection and mapping is extensive and time consuming. Nonetheless, systematic avalanche detection over large areas could help to generate more complete and up-to-date inventories (cadastres) necessary for validating avalanche forecasting and hazard mapping. In this study, we focused on automatically detecting avalanches and classifying them into release zones, tracks, and run-out zones based on $0.25 \mathrm{~m}$ near-infrared (NIR) ADS80-SH92 aerial imagery using an object-based image analysis (OBIA) approach. Our algorithm takes into account the brightness, the normalised difference vegetation index (NDVI), the normalised difference water index (NDWI), and its standard deviation $\left(\mathrm{SD}_{\mathrm{NDWI}}\right)$ to distinguish avalanches from other landsurface elements. Using normalised parameters allows applying this method across large areas. We trained the method by analysing the properties of snow avalanches at three $4 \mathrm{~km}^{-2}$ areas near Davos, Switzerland. We compared the results with manually mapped avalanche polygons and obtained a user's accuracy of $>0.9$ and a Cohen's kappa of 0.79-0.85. Testing the method for a larger area of $226.3 \mathrm{~km}^{-2}$, we estimated producer's and user's accuracies of 0.61 and 0.78 , respectively, with a Cohen's kappa of 0.67. Detected avalanches that overlapped with reference data by $>80 \%$ occurred randomly throughout the testing area, showing that our method avoids overfitting. Our method has potential for large-scale avalanche mapping, although further investigations into other
\end{abstract}

regions are desirable to verify the robustness of our selected thresholds and the transferability of the method.

\section{Introduction}

Snow avalanches are frequent and destructive mountain hazards, particularly during the winter and spring months. They are fast mass movements controlled by weather conditions, snowpack, and topography (Schweizer et al., 2003; Castebrunet et al., 2012). Avalanches can cause loss of lives, disrupt infrastructure, and bury buildings (Bründl et al., 2004; McClung and Schaerer, 2006; Eckerstorfer and Malnes, 2015).

Despite numerous efforts aimed at reducing the risk from avalanches, most fatalities in Europe occur during sporting activities, caused by avalanches triggered by the victims themselves (Techel et al., 2015). Past research indicates that poor decision-making and forecasting are the main causes of deadly avalanche accidents (Techel et al., 2015; McClung, 2016). Techel et al. (2015) stated that most destructive events occur on days when the snow-avalanche risk is very critical and the snowpack layer is weak. In total, 4750 people lost their lives in the European Alps between 1970 and 2015 (Techel et al., 2016); in the past 2 decades, avalanches have killed 461 people in the Swiss Alps alone (Fig. 1). Most fatal accidents have occurred in the cantons of Valais and Grison, which are the two largest in Switzerland and contain some of the most elevated areas in the Swiss Alps. Since 1946, avalanches in Switzerland have had the highest share of vic- 


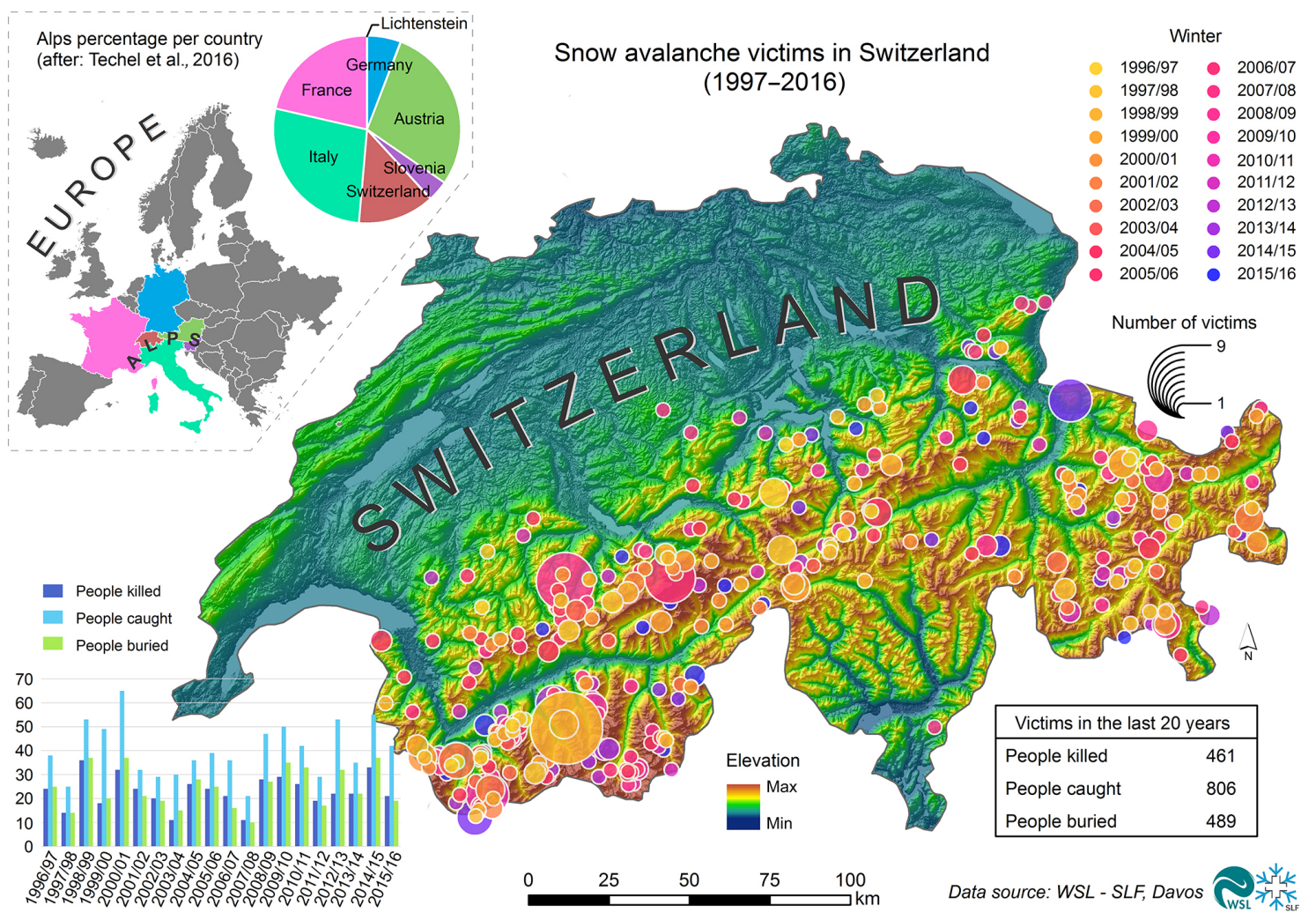

Figure 1. Snow-avalanche accidents with victims in Switzerland in the winters of 1996-1997 to 2015-2016 and the Alps percentage per country. People caught are those who had been caught by an avalanche but not totally buried. Data from WSL Institute for Snow and Avalanche Research SLF, Davos, Switzerland.

tims (37\%) compared to other natural hazards, including lightning (16\%), floods (12\%), windstorms (10\%), rockfalls $(8 \%)$, and landslides (7\%; Badoux et al., 2016).

Avalanches killed a total of 36 people in Switzerland in the winter of 1998-1999 (Fig. 1). Between 27 January and 25 February, 17 people died in villages and on roads, and material losses surpassed CHF 600 million. This catastrophic winter spurred an initiative aimed at improving avalanche safety and reducing concomitant losses (Wilhelm et al., 1999; Bründl et al., 2004). This initiative included establishing an information system for exchanging data between the WSL Institute for Snow and Avalanche Research SLF and local authorities, the development of hazard maps showing zones with high avalanche susceptibility (Bründl et al., 2004), and an increase in the artificial release of avalanches to decrease hazard levels. In this context, the need to document avalanches also increased to allow for learning from past accidents.

This initiative also showed clearly that regional-scale mapping of avalanches and identifying potential release zones is not only desirable but also essential for producing avalanche cadastre maps for quality-checking hazard mapping and forecasting (Bühler et al., 2015). To date, experts map most avalanches manually and at the national level mainly the geo- graphic coordinates of avalanches; rarely does this mapping involve detailed information about the avalanche geometry (Bühler et al., 2009). Hence, avalanche inventories are biased toward damaging events or those reported from accessible terrain, so that avalanches remain notoriously under-reported over larger regions. To more broadly collect information concerning avalanches, non-expert observers in Switzerland are now able to report sightings via an app (http://www. slf.ch/lawinenbulletin/rueckmeldung/index_EN), where they can enter the location and date of their observation. Optical remote sensing data, both airborne and satellite, offer coverage that is more systematic, and they are therefore increasingly used to track avalanches. Satellite images allow covering the same area with a time interval equal to one satellite orbit around the Earth. Airborne images can be acquired even more often, although in the winter season it is not preferable to continue the campaign due to financial constraints, because such images, which feature mostly only snow, are not convenient for any purposes other than the assessment of the risk of snow avalanches.

Automatic methods for detecting snow avalanches are still in the developing stage (Eckerstorfer et al., 2016), and different kinds of data, such as optical and radar images, and classification approaches are used to verify their suitability 

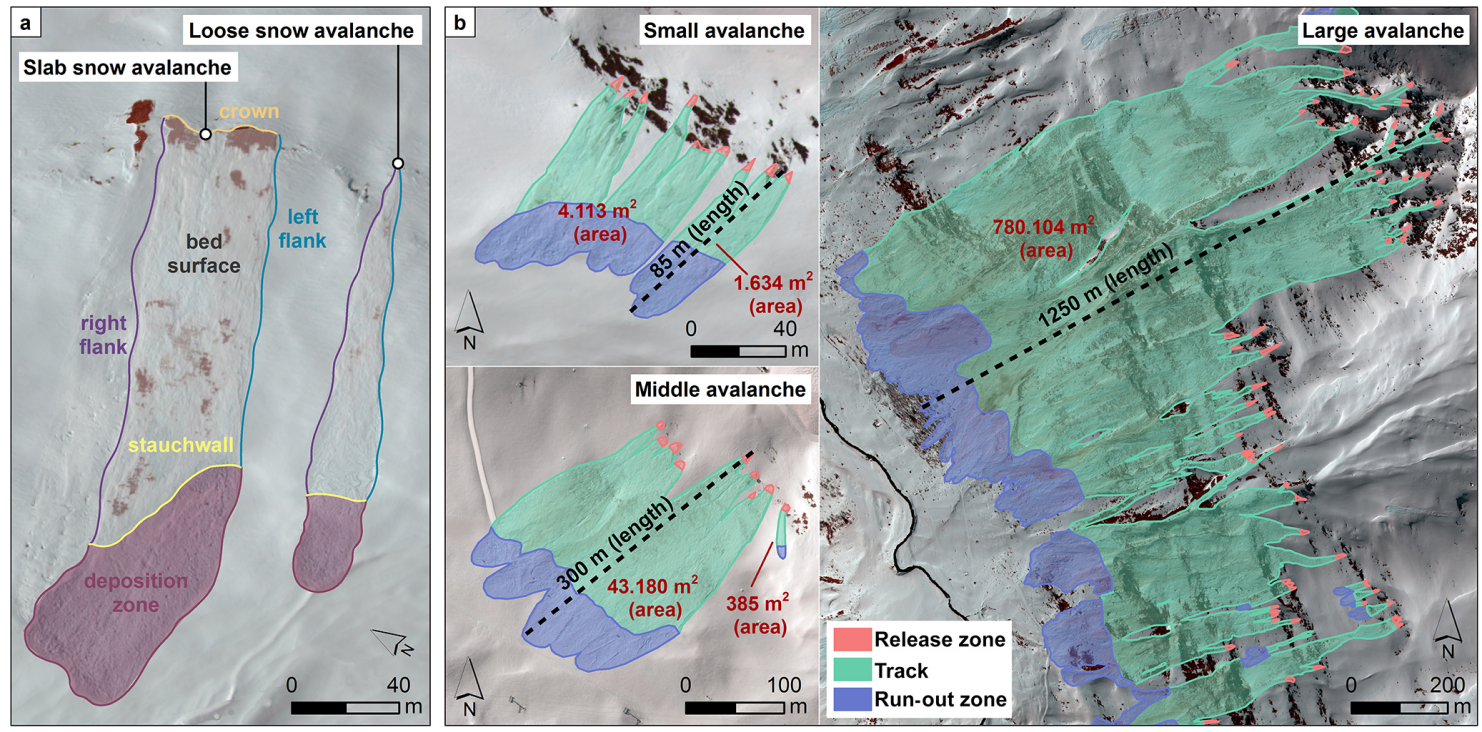

Figure 2. (a) Types of avalanche release: slab avalanches and loose avalanches, with marked avalanche parts (crown, bed surface, stauchwall, deposition zone, and right and left flanks; Schweizer et al., 2003); (b) avalanche classification with respect to length (small, middle, and large, in metres), with the avalanche area $\left(\mathrm{m}^{-2}\right)$ and marked parts of avalanches (release zone, track, and run-out zone). Data from WSL Institute for Snow and Avalanche Research SLF, Davos, Switzerland.

to track avalanche events. This motivated us to explore the use of near-infrared (NIR) aerial images and their derivatives for mapping avalanches over a large area in Switzerland, as well as investigating the topographic conditions of their occurrence. We propose an automatic method based on objectbased image analysis (OBIA) for detecting avalanche run-out zones, their tracks, and release areas. We test whether normalised indices of water and vegetation derived from aerial ADS80-SH92 images are suitable in this regard and introduce a simple method for roughly distinguishing these zones, because knowledge concerning potential release zones critically aids hazard assessments and run-out models. Our motivation was to develop an algorithm widely applicable to mountain regions; relying on image spectral properties alone (Lato et al., 2012) may limit such portability because objects may have a brightness similar to that of snow.

Most avalanches start on slopes with a median inclination of $39^{\circ}$ (Schweizer and Jamieson, 2001), on slopes most difficult for skiing. We distinguish two types of avalanche: loose avalanches start from a point and gradually increase in size as they move downslope, whereas slab avalanches involve the detachment of large planar packs of snow (Fig. 2; Schweizer et al., 2003; Bagli and Schweizer, 2009). Path length defines whether an avalanche is small $(10-100 \mathrm{~m})$, medium (100-1000 m), or large (>1000 m; Eckerstorfer et al., 2016). Most large avalanches are slab avalanches because to bring down a large amount of snow, planar snow detachment is necessary (Fig. 2); however, new smaller loose snow avalanches may occur and overlap with the previous one, thus complicating their detection in the field. In terms of avalanche deposit area, we distinguish between large deposits $\left(>2000 \mathrm{~m}^{-2}\right)$, small deposits $\left(100-2000 \mathrm{~m}^{-2}\right)$, and very small deposits $\left(<100 \mathrm{~m}^{-2}\right.$; Bühler et al., 2009). Every avalanche has a release zone (a part where the avalanche is triggered), a track (a part where the snow is transported down the slope), and a run-out, or deposition, zone (Fig. 2).

\section{Previous work}

Most previous work devoted to mapping avalanches from optical remote sensing data has focused on delineating run-out zones. The idea of using OBIA for detecting avalanches has been used in conjunction with brightness information from aerial images and local slope data from digital elevation models (DEMs) and with numerical modelling (Bühler et al., 2009), whereas others have used only the spectral information of aerial and satellite images (Lato et al., 2012). Bühler et al. (2009) mapped snow-avalanche deposits from ADS40 $20 \mathrm{~cm}$ aerial images, which they resampled to $1 \mathrm{~m}$ and then combined with $25 \mathrm{~m}$ elevation data. They used the numerical simulation tool RAMMS (Rapid Mass Movement Simulation; Christen et al., 2010) to identify possible avalanches and excluded slopes $>35^{\circ}$ from the run-out calculation, assuming that these slopes could not accumulate snow-avalanche debris. They also worked with spectral thresholds to exclude snow-free areas. To separate rough avalanche debris from surrounding smooth and undisturbed snow, they used the normalised difference angle index (NDAI), evaluated from nadir and backwards NIR bands. They computed the NDAI difference between neighbouring pixels with a grey-level co- 


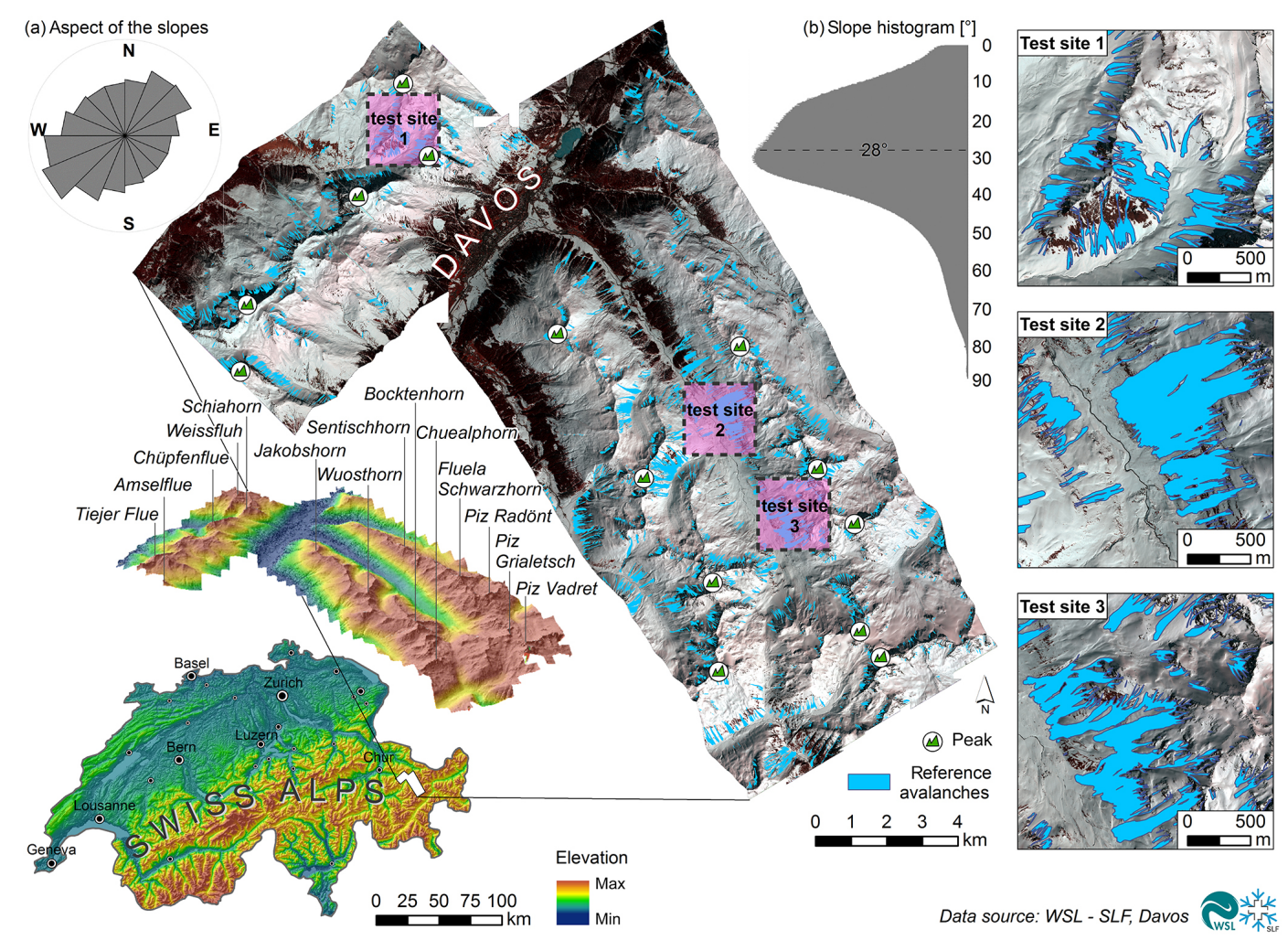

Figure 3. Topographic setting of research area and ADS 80 NIR aerial images with test sites (1-3) and digitally mapped snow avalanches; blue marks snow-avalanche polygons. Inset histograms show (a) distribution of local slope and (b) the main slope aspect.

occurrence matrix (GLCM), which represents the distribution of pixel values at a given offset, and found that the thresholding of an entropy measure evaluated via the GLCM achieved the best separability of rough and smooth snow. Ski lifts and other objects characterised by a similar entropy were removed using OBIA. The estimated accuracy of this method in terms of the fraction of correctly detected avalanche deposits was $94 \%$, and the producer's accuracy was $87 \%$. Lato et al. (2012) applied OBIA for detecting avalanche deposits from panchromatic images only. They tested their algorithm with QuickBird images in Norway and aerial ADS40 images in Switzerland, relying on six variables (i.e. GLCM entropy, GLCM dissimilarity, brightness, contrast, similarity, and neighbour distance) in their procedure, in which segments failing to meet the OBIA assumptions were sequentially discarded. They started by eliminating dark regions from brightness data before detecting rough snow with edge contrasts. The similarity filter and density helped to remove isolated pixels and small objects, respectively. Finally, the neighbour distance helped to fill gaps inside the extracted snow-avalanche deposits. The user's and producer's accuracies of that classification were both $>90 \%$. Both these studies (Bühler et al., 2009; Lato et al., 2012) regarded OBIA as suitable for detecting snow-avalanche deposits because it considers the spatial relation of the analysed segments in addition to their spectral properties.

Larsen et al. (2013) suggested an approach for optical QuickBird imagery using directional filters evaluated based on image texture classification (Varma and Zisserman, 2004) to distinguish avalanches from other objects. They assumed that avalanches have a linear texture pattern coinciding with the local hillslope aspect. Similar to other strategies, their classification took into account neighbouring pixels, while parameters such as area, area-perimeter ratio, aspect direction difference, co-occurrence mean, correlation, and entropy helped to exclude misclassified instances. Based on a visual comparison, the authors concluded that their classification was acceptable, allowing the detection of many fresh avalanches with a low number of false alarms (Larsen et al., 2013). They pointed out, however, that some of the detected avalanches were split into parts; they therefore recommended additional processing to reconnect those fragments.

Eckerstorfer and Malnes (2015) manually detected avalanche debris based on its higher backscatter contrast, compared to the surrounding undisturbed snow cover, in RADARSAT-2 ultra-fine synthetic-aperture radar (SAR) imagery. They assumed that avalanches are tongue-shaped features with high surface roughness and higher snow density than surrounding terrain. Surface roughness and snow den- 


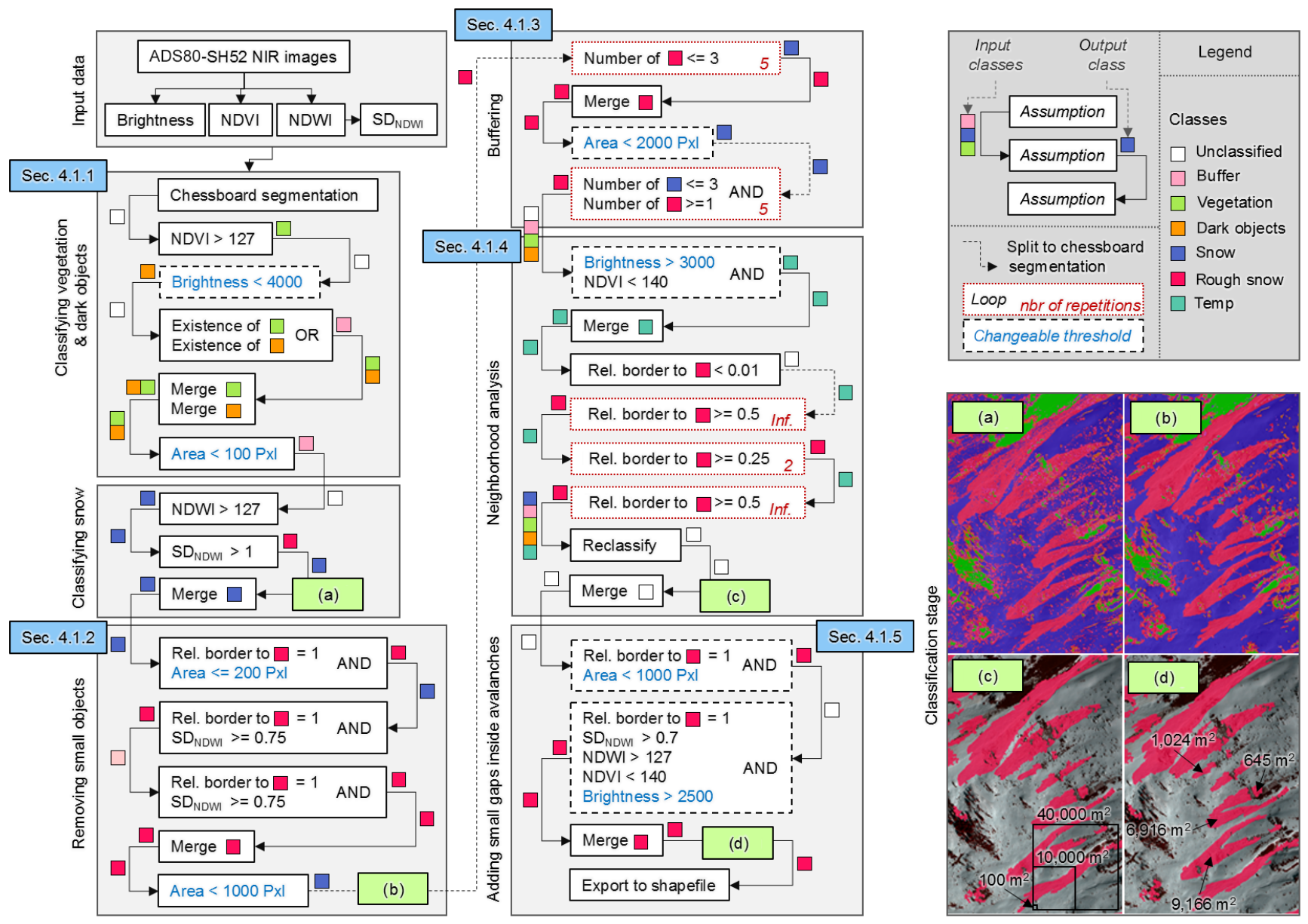

Figure 4. Workflow for classifying snow avalanches with object-based image analysis (OBIA). The white boxes indicate the classification; dashed outlines feature the number of reiterations (red), and the local decision boundaries show change (blue). The colours of squares are coded to the input and output class in each step. Panels (a-d) represent visual results of the sub-step classification. The squares and numbers on (c) and (d) represent a sample segment size. Section 4.1.1-4.1.5 in the figure are the numbers of subchapters in the article.

sity were determined from backscatter, which increased in cases of higher surface roughness, and absorption, which increased for denser snow, respectively. They found that release zones and tracks were mostly difficult to detect. In a similar context, the automatic method by Vickers et al. (2016) evaluates backscatter in $50 \times 50$ pixel regions of Sentinel-1A images, subsequently masking out areas with a predicted zero probability of snow-avalanche occurrence; pixels with a DEM-derived local slope of $>35^{\circ}$ were ignored. From the test pixels, they selected those with a backscatter difference above a specified threshold. Randomly selected pairs of pixels gave a total dissimilarity of pixels and class representatives for a $K$-mean clustering with two classes, i.e. avalanche or not avalanche. The producer's accuracy of this algorithm was 0.6 , and the authors highlighted its potential for avalanche monitoring despite having to mask out large amounts of data. Bühler et al. (2016) tested an unmanned aerial vehicle (UAV) that allows for fast, repeatable, flexible, and cost-efficient measurements of snow depths in alpine terrain, possibly generating digital surface models (DSMs) of homogenous snow surfaces (Bühler et al., 2017). Legal regulations in Switzerland and elsewhere currently limit broad coverage of UAV imagery, however.

\section{Study area and data}

Our study area is centred around Davos, in the Swiss canton of Grisons; the area has alpine relief, with the highest local peak at Schwarzhorn (3146 m a.s.l.; Fig. 3). Many slopes in this area exceed $28^{\circ}$ and have dominant north-eastern and south-western aspects (Fig. 3). We used $0.25 \mathrm{~m}$ resolution NIR aerial images in conjunction with abundant avalanche information acquired via a ADS80-SH92 large-format digital push-broom sensor (Leica Geosystems AG, Heerbrugg, Switzerland; Bühler et al., 2009) at the end of the 20122013 winter season. The sensor recorded information with 

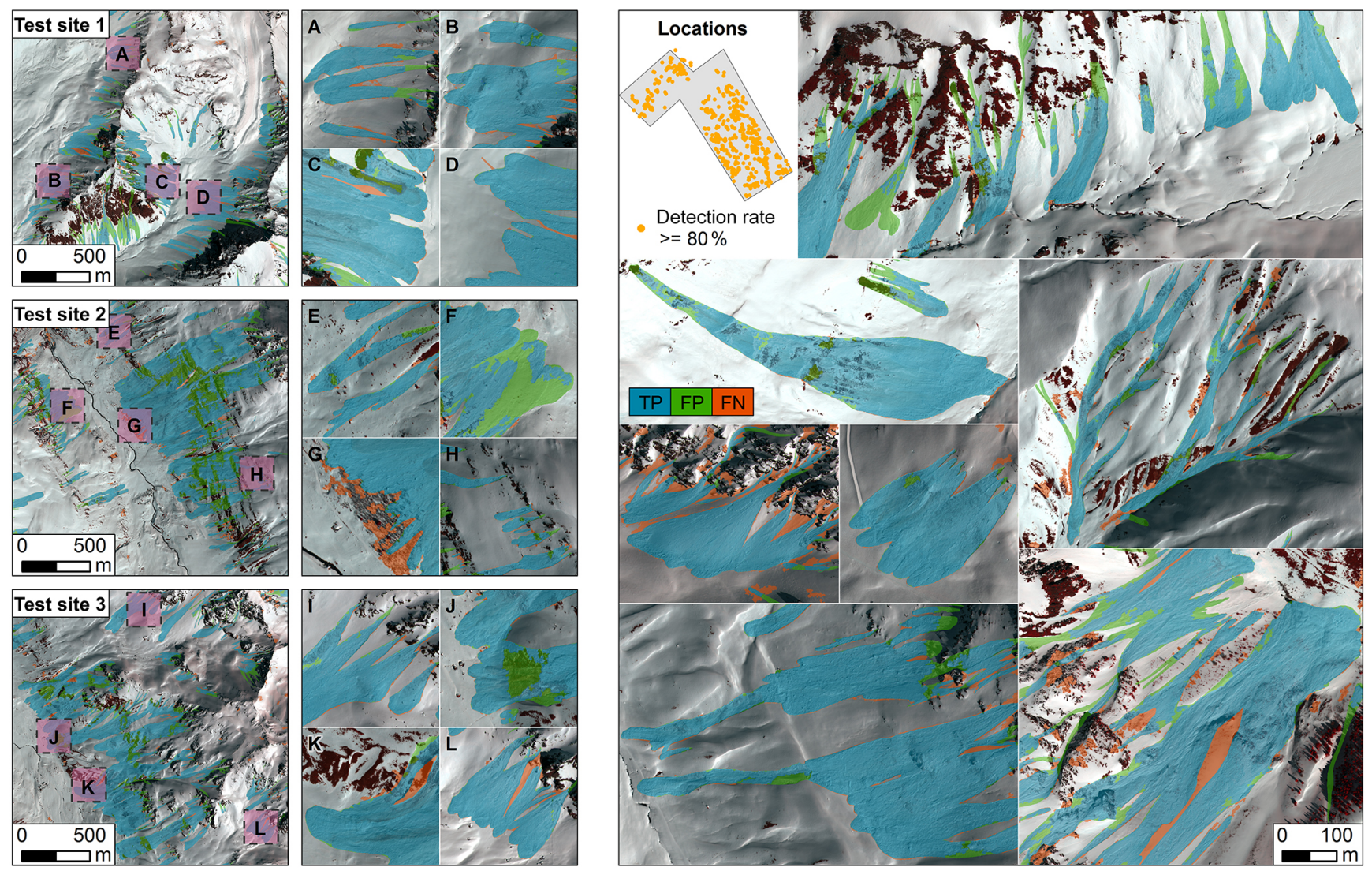

Figure 5. Accuracy assessment of OBIA classification for training (test sites 1-3) and testing data together; avalanche debris are shaded with orange colour where they were detected with an accuracy $\geqq 80 \%$. TP is true positive; FP is false positive; FN is false negative.

five spectral bands: panchromatic, blue, green, red, and NIR (Bühler et al., 2009). We have data for the area for more than five time slices starting from the winter of 2007-2008 and continuing from 2011-2012 to 2015-2016. The images we used were taken at the end of the winter of 2012-2013, where the highest expected snow depths were between 2000 and $3000 \mathrm{~m}$ a.s.l. and covered $\sim 226 \mathrm{~km}^{-2}$.

\section{Methods}

We introduce an automatic method for mapping release zones, tracks, and run-out zones of avalanches using NIR $0.25 \mathrm{~m}$ aerial images (Sect. 4.1). We compare the automatic classification with manually digitised reference data and estimated the accuracy of detecting snow avalanches with confusion matrices (Sect. 4.2, 5.1). We also investigated the topographical conditions in which most mapped avalanches occurred (Sect. 5.3), using two approaches for visualising avalanche density (Sect. 5.4). We also propose a probability approach to representing release and run-out zones of avalanches and the automatic classification of snowavalanche parts (Sect. 5.5.).

\subsection{Automatic OBIA snow-avalanche classification}

We implement a multi-step OBIA approach for detecting avalanches in eCognition Developer 9.1.1 software (Fig. 4). As input for the classification, we used the green, red, and NIR bands and computed from these the normalised difference vegetation index $\left(\mathrm{NDVI}=\rho_{\mathrm{NIR}}-\rho_{\mathrm{Red}} / \rho_{\mathrm{NIR}}+\right.$ $\rho_{\text {Red }}$; Townshend and Justice, 1986) and the normalised difference water index $\left(\mathrm{NDWI}=\rho_{\text {Green }}-\rho_{\mathrm{NIR}} / \rho_{\text {Green }}+\rho_{\mathrm{NIR}}\right.$; McFeeters, 1996) and its standard deviation in a $5 \times 5$ kernel ( $\left.\mathrm{SD}_{\mathrm{NDWI}}\right)$. We derived brightness as the mean of the green, red, and NIR bands to classify "dark objects" such as rivers, rocks, and buildings. The NDVI helps to classify trees, bushes, and other types of vegetation, whereas the NDWI

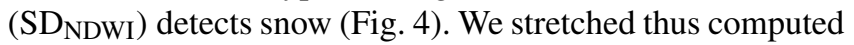
NDVI and NDWI data into an interval of $[0,255]$.

\subsubsection{Classifying vegetation, dark objects, and snow}

In a first step, we segmented the data using a chessboard segmentation algorithm in eCognition 9.1.1, assigning a standalone segment to each pixel (Fig. 4). We used the pixel NDVI and brightness values of snow-free areas to classify vegetation and dark objects. We classified vegetation as having pos- 

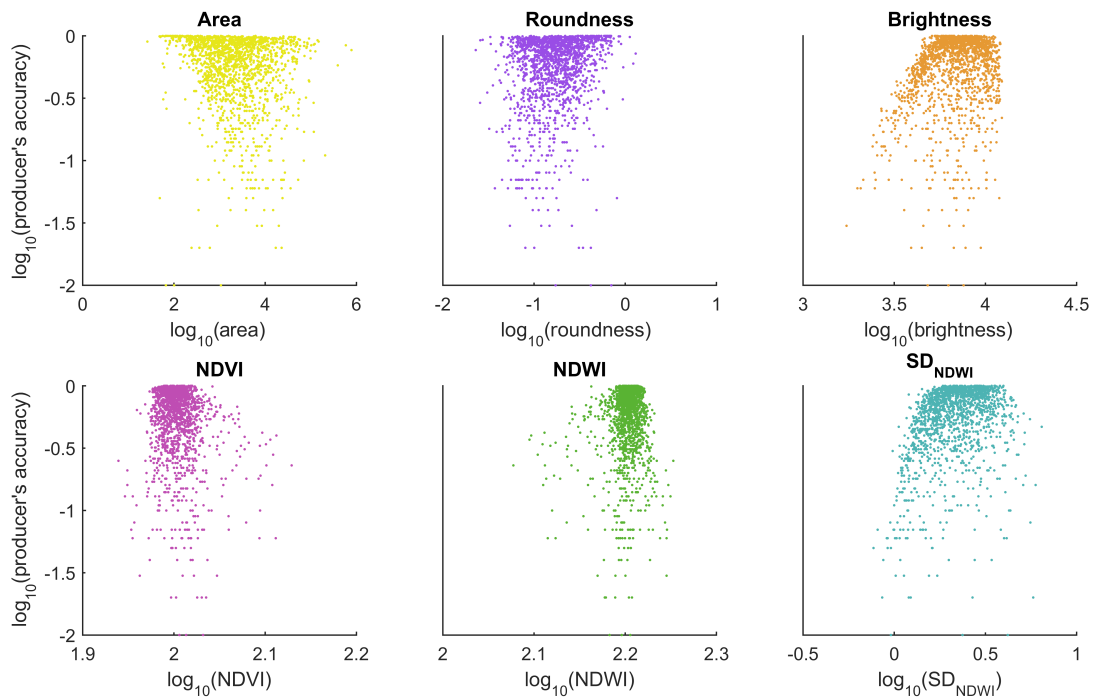

Figure 6. The role of avalanche area, roundness, brightness, normalised difference vegetation index (NDVI), normalised difference water index (NDWI), and standard deviation of normalised difference water index (SDNDWI) in the estimated accuracy when detecting snow avalanches with our OBIA approach.
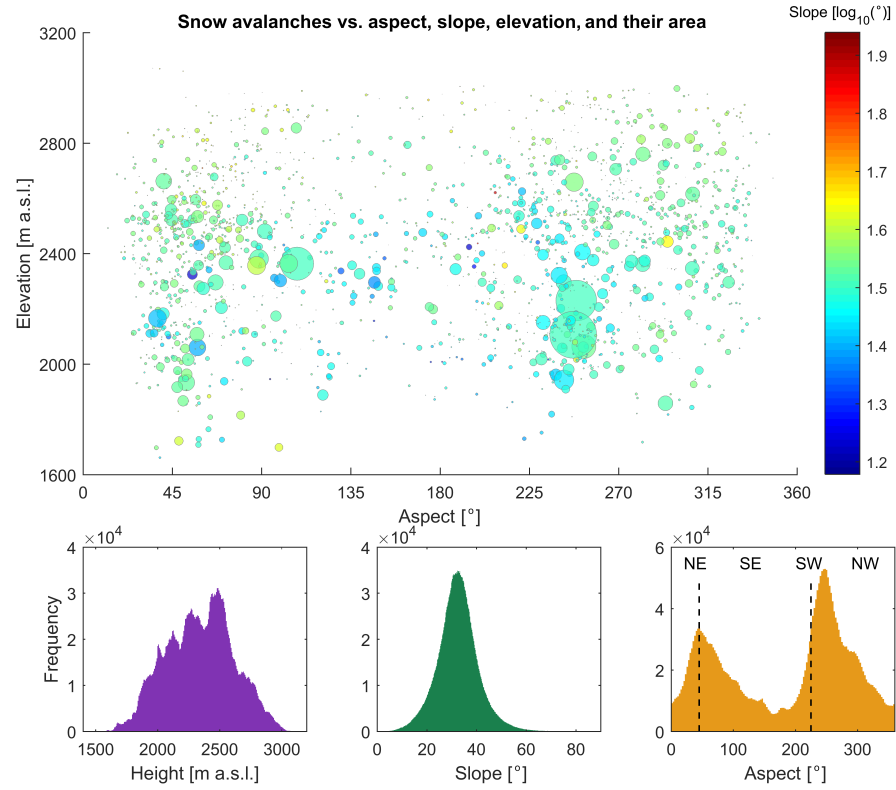

Figure 7. Elevation, slope, and aspect of mapped reference avalanches with marked north-east and south-west directions in the winter of 2012-2013. The values presented for every single avalanche represent the mean value of each pixel contained inside the reference avalanche polygon. Bubble size on the scatter plot is scaled to avalanche area.

itive NDVI values (corresponding to $>127$ in the stretched range) and dark objects as those with a brightness of $<4000$ (Fig. 4). Because pixels on the border of vegetation and dark objects have mixed values, we assigned them to a separate "buffer" class by reclassifying every pixel that shared a border with either vegetation or dark objects (existence of vegetation or existence of dark objects; Fig. 4) and then excluded this buffer class from further analysis. We merged all seg- ments classified as vegetation and dark objects and treated all segment areas $<6.25 \mathrm{~m}^{-2}(<100$ pixels; Fig. 4) as too small to buffer or divert an avalanche, and we included these segments as potential avalanche areas. All the size thresholds in our OBIA workflow were set to the resolution of the data and the size of the mapped avalanches. To select the best size of segments we ran a sensitivity analysis using a trial-and-error approach in the eCognition software, visually checking the 

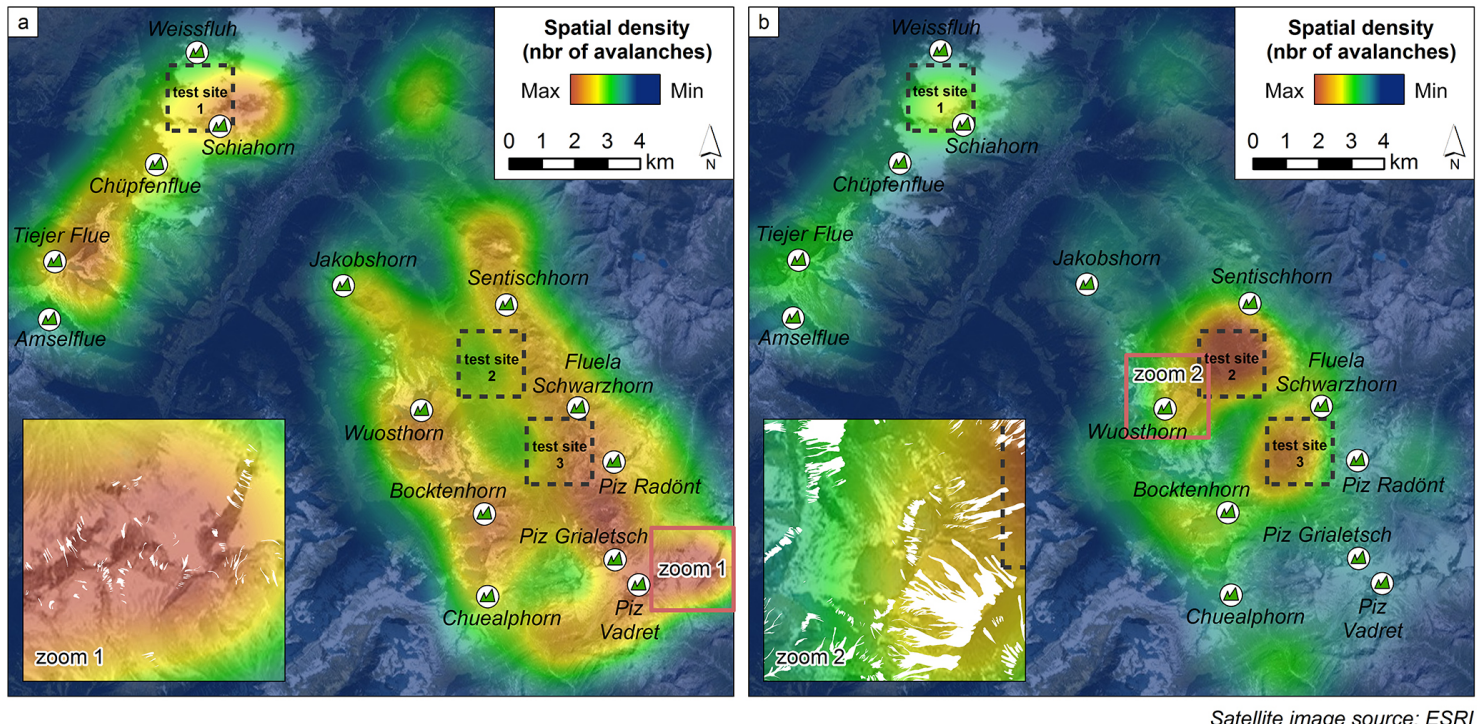

Figure 8. Density maps showing the clustering of avalanches in the winter of 2012-2013 with respect to their (a) quantity and (b) size. The insets (zoom 1 and zoom 2) show the density maps with respect to manually classified reference snow-avalanche polygons shown in white. Outlined test sites 1-3 are the test sites that were used for developing our OBIA algorithm.

results for various thresholds. Similarly, we classified pixels with a positive NDWI ( $>127$ for stretched data) as snow and pixels with an additional roughness contrast $\left(\mathrm{SD}_{\mathrm{NDWI}}>1\right)$ as "rough snow", representing avalanches.

\subsubsection{Removing small objects that do not represent avalanches}

We then merged all snow pixels and reclassified rough snow segments $<12.5 \mathrm{~m}^{-2}$ ( $<200$ pixels, Fig. 4$)$. We thus included many pixels as parts of avalanche deposits that in the previous step had escaped being classified as rough snow because of too-low $\mathrm{SD}_{\mathrm{NDWI}}$ values. We set a maximum area of segment to be reclassified as rough snow to avoid including large, but smooth, areas inside avalanches that are not avalanche debris. After comparing the segment values with their visual representation, we observed that smooth areas inside of avalanche debris were areas of flow separation. We similarly assumed that the boundary of rough snow should be equal to 1 for the snow and buffer classes, so that the segment lies completely within these two classes; we used a $\mathrm{SD}_{\mathrm{NDWI}}$ of $\geqq 0.75$ to include the segment as rough snow. The thresholding value of the $\mathrm{SD}_{\mathrm{NDWI}}$ came from the most pronounced changes in the histogram data. We then merged the segments into the rough snow class except for areas $<62.5 \mathrm{~m}^{-2}$ (area $<1000$ pixels; Fig. 4), assuming that these are too small to represent an avalanche. We chose this area threshold based on visual checks and a trial-and-error sensitivity analysis in the eCognition software.

\subsubsection{Buffering}

In further steps, we split all segments from the rough snow class into smaller pieces to reduce their complex shapes with bigger and more compact parts connected to neighbours by only a few pixels. In most cases, only some of these complex shapes represented an avalanche, whereas the remainder was rougher snow due to vegetation effects. To simplify these shapes into separate parts, we used buffering to reclassify pixels from the rough snow class as snow that had less than four neighbours classified as rough snow (Fig. 4), repeating this step for both the snow and rough snow classes. To avoid undue growth of spurious pixels, we narrowed down the process to only pixels adjacent to at least one pixel classified as rough snow (Fig. 4).

\subsubsection{Neighbourhood analysis}

At this stage, our classification still contained many misclassified parts of avalanches containing effects of vegetation, soil, or rocks. To assign these parts to the rough snow class, we first reclassified all pixels from unclassified, buffer, vegetation, and dark objects with brightness $>3000$ and NDVI $<140$ into a new "temp" class (Fig. 4). The brightness thresholds we derived from a sensitivity analysis, in analogy to previous steps (Fig. 6). After merging the segments into the temp class, we reclassified all segments bordering rough snow $<0.01$ into "unclassified" and discarded these from the analysis. For the remaining segments, after returning to chessboard segmentation to allow us to once again operate on single pixels, we reclassified all pixels from the temp class sharing a border with rough snow with at least two 
Evaluating the probability

1. Resampling DSM into $0.25 \mathrm{~m}$

2. Clipping DSM to avalanche boundaries

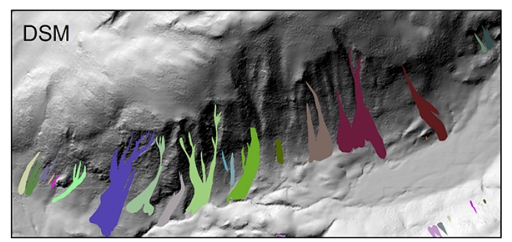

3. Evaluating the probability using formula: probability $=$ value $_{\mathrm{DSM}}-\mathrm{min} / \mathrm{max}-\min$

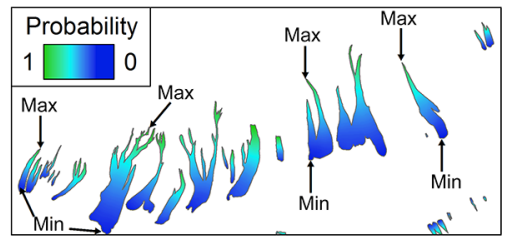

4. Classifying the probability into three classes: 0-0.3 (run-out zone)

$0.3-0.8$ (track)

0.8-1 (release zone)

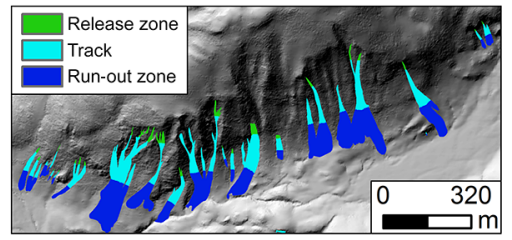

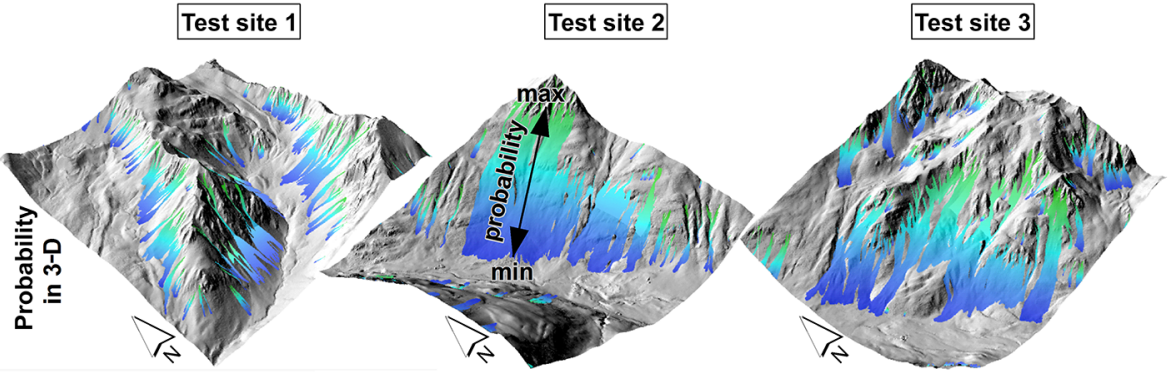
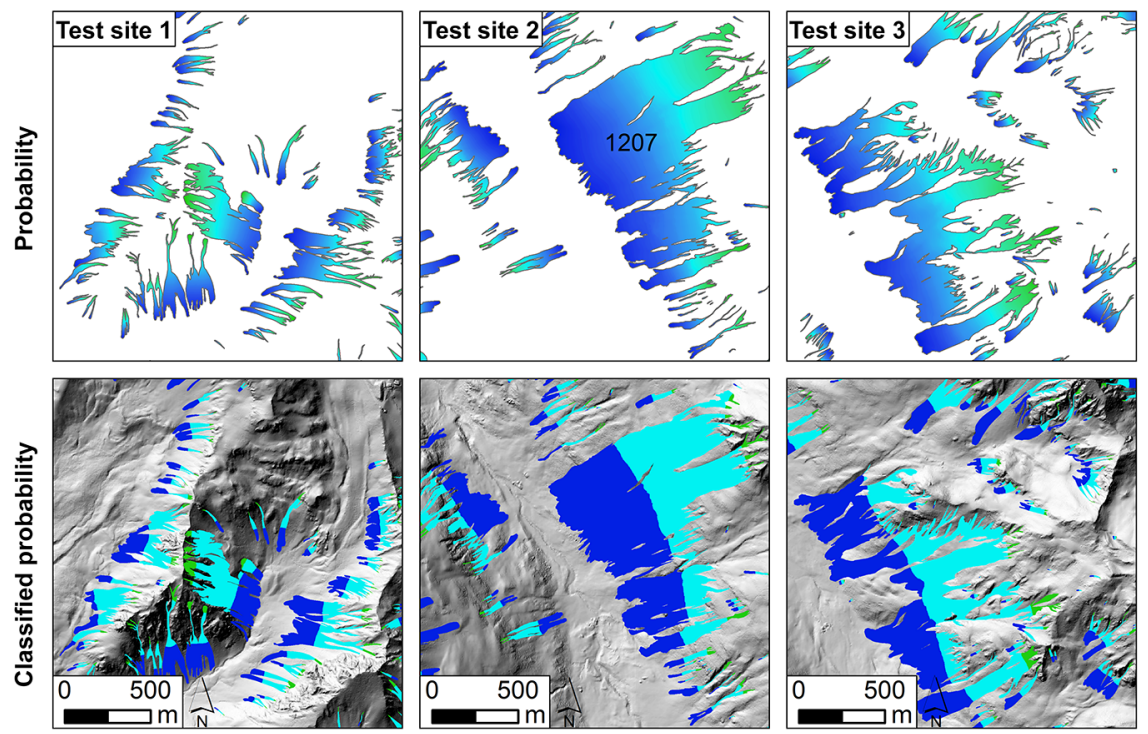

Figure 9. Estimating the probability of an avalanche release area. A probability close to 1 indicates a pixel representing a release zone, whereas a probability close to 0 indicates a run-out zone in automatically classified data.

pixels (Fig. 4) iteratively. Because every pixel had only four neighbours, the assumption concerning two pixel neighbours stopped the infinite loop after only a few repetitions. To allow the inclusion of additional pixels, we therefore decreased the threshold on the relative border to $\geqq 0.25$ and performed the process twice more to allow more segments that were sharing the boundary with at least one rough snow segment to be included in the rough snow class. We did not run this process iteratively because it would have reclassified all pixels assigned as temp, and our aim was to increase only compact rough snow areas. Next, we once more applied an infinite loop regarding the relative border to rough snow of $\geqq 0.5$ to increase previously detected avalanches. These steps were crucial in closing areas inside the avalanches that, due to the values of brightness and NDVI, were not assigned in the previous steps to the rough snow class.

\subsubsection{Adding small gaps inside avalanches}

Finally, we focused on filling gaps inside the detected snow avalanches. We reclassified and merged gaps into avalanches by verifying their geometrical relation to rough snow. After checking the layer statistics for every segment, we built an assumption that if a segment was completely within the rough snow and its area was $<1000$ pixels $\left(62.5 \mathrm{~m}^{-2}\right)$, it was to be automatically reclassified into rough snow (relative border to rough snow $=1$ and area $<1000$ pixels; Fig. 4). Segments with $>1000$ pixels reclassified into rough snow were expected to fulfil additional rules concerning their roughness, brightness, occurrence of snow, and vegetation, because they may have represented a convex-upward form that could stay intact during avalanching. Only segments with a high snow roughness that did not represent vegetation and were not too dark were added to the rough snow class (relative border to rough snow $=1 ; \mathrm{SD}_{\mathrm{NDWI}}>0.7, \mathrm{NDWI}>127, \mathrm{NDVI}<140$, and brightness $>2500$; Fig. 4). The threshold selected for brightness is lower than in previous steps because some parts of snow avalanches were a little darker; hence using the same threshold would cause unwanted misclassifications. We exported all extracted snow avalanches into polygon shapefiles and compared these visually and quantitatively with manually mapped reference data. The visual interpretation was important for verifying the distribution of errors and the completeness of classified avalanches. 


\subsection{Generating reference data}

We created reference data by manually digitising avalanches from the images in ArcMap 10.3 software at scales between $1: 800$ and $1: 1500$, depending on the complexity of the avalanche debris. Manually digitising each area affected by the avalanche was necessary because avalanches occur only during the winter season when snow cover occurs, making them temporal events; the marks of their existence disappear when the snow cover melts, and therefore no complete reference data are available. Avalanches that were overlapping or bordering others were counted as one; some polygons contained several smaller avalanches that had occurred in succession. In most cases, it was impossible to assess their relative sequence (Fig. 3). We mapped a total of 2200 avalanche polygons for the data acquired in the winter of 2012-2013, covering $13.6 \mathrm{~km}^{-2}$ of avalanche terrain or $6 \%$ of the study area. These reference data are available online (https://uni-potsdam.maps.arcgis.com/apps/Cascade/ index.html?appid=3b5ac4491b59480c8c6016139f285e88), and we used them to estimate several performance metrics, including Type I, Type II, and total errors (Sithole and Vosselman, 2004); overall, user's, and producer's accuracies (Congalton, 1991); Cohen's kappa (Cohen, 1960); and F score. We arbitrarily selected three $4 \mathrm{~km}^{-2}$ training sites (Fig. 3) for our OBIA algorithm and reported these eight performance metrics for a larger $\left(226.3 \mathrm{~km}^{-2}\right)$ test area.

\section{Results}

\subsection{Estimated accuracy}

Our algorithm classified $10.7 \mathrm{~km}^{-2}$ as avalanche debris, which is $78.7 \%$ of the total area of the reference data mapped for the winter of 2012-2013. Overall, 1648 out of 2200 avalanches were correctly identified; 1126 were detected in terms of more than half their area, and for 615 avalanches this detection rate was $>80 \%$. These classified avalanches were spread out evenly throughout the study area (Fig. 5). Visual checks of the classification indicated that the run-out zones were detected most reliably, whereas the release zones were the most problematic. Tracks were detected mostly correctly where small patches of vegetation or soil were near or in the avalanche tracks. The highest estimated precision in detecting avalanche boundaries was in run-out zones adjacent to smooth snow or bare slopes. In some locations, a clear distinction between avalanche debris and smooth snow was difficult, especially for older deposits or snow drifts. Fresh avalanches were also detected with higher accuracy than older and blurred ones.

The performance metrics estimated for our training sites were overall and user's accuracies of $>0.9$ and a Cohen's kappa of $>0.8$ (Table 1). The accuracy for the testing area yielded lower performance metrics, with a user's accuracy of
0.78 . The producer's accuracy showed that $61 \%$ of the total avalanche area in the tested data was correctly identified. The overall Type II error was very low, indicating that few objects were falsely classified as avalanches, whereas the high Type I error showed that many, mostly old, snow avalanches remained undetected.

\subsection{Influence of variables used for classification accuracy}

We checked how brightness, NDVI, NDWI, and SD $_{\text {NDWI }}$ derivatives evaluated for each classified avalanche affected the producer's accuracy. For each avalanche, we computed the mean for each derivative map using the values of all the pixels inside the avalanche. We found that avalanches that were extracted with the highest accuracy were generally also brighter (Fig. 6); an increase in producer's accuracy occurred with an increase in the mean brightness of avalanche. A similar, but weaker, correlation held for SDNDWI. Neither NDVI nor NDWI had much of an influence on the classification accuracy. In addition, we verified whether an avalanche's shape (roundness) and size (area) affected the detection rate. Similarly to NDVI and NDWI, however, we did not find any dependence (Fig. 6).

\subsection{Topographic factors favourable for snow avalanches}

We further analysed the topographic settings of the mapped avalanches. Most avalanches (1422 out of 2200) occurred between 1900 and $2600 \mathrm{~m}$ a.s.l., with a mode of approximately $2400 \mathrm{~m}$ a.s.1., on slopes that were $20-40^{\circ}$ (Fig. 7). Our results show that avalanches were also detected on slopes steeper than $35^{\circ}$, thus highlighting the limits of detection methods using arbitrary slope thresholds (Bühler et al., 2009; Vickers et al., 2016). Out of 193, 139 of the highest-lying avalanches (>2800 ma.s.1.) were small or very small, according to the nomenclature of Bühler et al. (2009) and occurred in the southern part of our research area. Although the largest avalanches occurred below $2400 \mathrm{~m}$ a.s.l., most affected north-eastern and the south-western slopes, thus mimicking the major aspects of the mountain ranges (Fig. 3).

\subsection{Density of avalanches}

Using the reference data for the winter of 2012-2013, we further analysed where most of the avalanches occurred. We computed centroid locations for each avalanche polygon, and estimated their spatial density using the kernel density function in ArcGIS 10.3, with both point- and area-weighted inputs in a $2 \mathrm{~km}$ radius. We produced two maps because, due to the occurrence of multiple avalanches, our input centroids did not represent the total number of events. Consequently, a point-weighted map could have underestimated the real avalanche density, whereas an area-weighted map avoids this issue. The selected $2 \mathrm{~km}$ size of the bandwidth 
Table 1. Performance metrics estimated for $4 \mathrm{~km}^{-2}$ training sites 1-3 (see Figs. 3 and 5) and for the entire study area covering $226.3 \mathrm{~km}{ }^{-2}$.

\begin{tabular}{lrrrrrrrr}
\hline & $\begin{array}{r}\text { Type I } \\
\text { error }\end{array}$ & $\begin{array}{r}\text { Type II } \\
\text { error }\end{array}$ & $\begin{array}{r}\text { Total } \\
\text { error }\end{array}$ & $\begin{array}{r}\text { Overall } \\
\text { accuracy }\end{array}$ & $\begin{array}{r}\text { Producer's } \\
\text { accuracy }\end{array}$ & $\begin{array}{r}\text { User's } \\
\text { accuracy }\end{array}$ & $\begin{array}{r}\text { Cohen's } \\
\text { kappa }\end{array}$ & $F$ score \\
\hline Site 1 & 0.23 & 0.01 & 0.05 & 0.95 & 0.77 & 0.91 & 0.81 & 0.83 \\
Site 2 & 0.23 & 0.02 & 0.08 & 0.92 & 0.77 & 0.92 & 0.79 & 0.84 \\
Site 3 & 0.16 & 0.02 & 0.05 & 0.95 & 0.84 & 0.93 & 0.85 & 0.88 \\
\hline Total & 0.39 & 0.01 & 0.03 & 0.97 & 0.61 & 0.78 & 0.67 & 0.69 \\
\hline
\end{tabular}

was large enough to avoid reproducing the pattern of input avalanche centroids and small enough to reduce the smoothing of the point information. We found that avalanches clustered largely in the south-eastern part of the study area (125 smaller avalanches in inset in Fig. 8a, of which 104 were $<2000 \mathrm{~m}^{-2}$ ). The area-weighted spatial density of avalanches was highest on the slopes of Fluela Schwarzhorn, Sentischhorn, and Wuosthorn. The biggest avalanche was $\sim 390000 \mathrm{~m}^{-2}$ (Fig. 8b).

\subsection{Automatic classification of snow-avalanche zones}

Automatic delineation of release and run-out zones from remote sensing data can be conducted by using the elevation range in each avalanche polygon. The simplest approach uses flow length, which is the distance along the flow path inside the avalanche, as an indicator in evaluating the probability of the release and the run-out zones. We suggest using the elevation values in an approach that allows exposure of local terrain height differences in the probability map. For example, applying the elevation for a release zone that occurs on very steep slopes determines that the highest probability will be represented only for a very small area, whereas on gentle slopes the same probability will occur over a larger area because the elevation differences are smaller than those of steep slopes.

We used a $2 \mathrm{~m}$ resolution DSM derived from stereo matching of aerial ADS images to automatically detect release zones, tracks, and run-out zones. Because we generated our reference data in the same resolution as the aerial images, we first resampled the DSM to $0.25 \mathrm{~m}$ resolution (Fig. 9). We then used the reference data to clip the DSM to each avalanche polygon using a key ID; the DSMs acquired in this way were used to compute the probability of representing individual parts of an avalanche. We assumed that the maximum (minimum) elevation on which the snow avalanche occurs has the highest probability of being a release (run-out) zone. We estimated the probability by normalising elevation data (Fig. 9) by subtracting the minimum value of DSM inside this avalanche from DSM pixel value and dividing by the difference between the maximum and the minimum values inside each avalanche. Such stretching returns a 0-1 probability map for release areas. The thresholds in classifying the track are relative; we applied $0.3-0.8$ as the thresholds because most of our 2200 manually digitised polygons represented loose snow avalanches where the release zone constituted a small part and the run-out zone compared to the track constituted an even smaller part. When dealing only with loose avalanches, the upper threshold for release areas may be set to a probability equal to 0.9 . The selected thresholds worked well for single avalanches, where both the release zone and the run-out zone were assigned correctly (Fig. 9). For avalanches with more complex shape and multiple avalanche arms (e.g. avalanche 1207 on test site 2; Fig. 9), however, the release zones remain undetected, with the exception of only the highest-lying release areas.

\section{Discussion}

Automatic mapping of avalanches is crucial in mountainous regions to delineate susceptible areas and to produce cadastres for validating avalanche forecasting and hazard maps. Previous studies have demonstrated that a combination of aerial images and digital elevation models (Bühler et al., 2009), or aerial (Lato et al., 2012) and satellite (Larsen et al., 2013), or SAR images (Vickers et al., 2016) favours the automatic detection of snow-avalanche deposits; however, identifying release zones and tracks remains challenging. We propose an OBIA algorithm that tracks release zones, tracks, and run-out zones in NIR images. We recommend using normalised derivatives NDWI and NDVI, instead of brightness, for classifying water (snow) and vegetation, respectively, because the thresholds allowing for classification of water and vegetation in these indices are stable (around zero) and widely applicable. Our approach expands upon previous work, as we have considered potential avalanches in snow-covered areas only (NDWI>127), whereas others have used brightness thresholds (Lato et al., 2012), with the pitfall of including other objects with similar values to those of snow. Rivers and lakes have similar values of NDWI but are less bright than snow, and we therefore combined NDWI and brightness in our model. We suggest using $\mathrm{SD}_{\mathrm{NDWI}}$ to trace rough snow or avalanche debris. Estimated accuracy was high for detecting avalanches using the test data, and the random spread of avalanches detected with high accuracy through the whole research area suggests that the assump- 
tions in our approach are broad enough to be applied for a large area.

To increase the accuracy of true positives in detecting release zones in our classification, a customised threshold for every data tile can be applied. In any case, we focused on developing a method that is transferable and works well for a greater area. We also wanted to test the usability of the image derivatives and verify how much information we could obtain from them when detecting snow avalanches. We therefore did not change any parameter or its threshold when applying the algorithm for other data tiles (Lato et al., 2012). We implemented several steps that allowed the classification of different avalanche scenarios that occur in diverse topographic conditions, such as single vs. multiple avalanche, small vs. large avalanche, avalanche revealing the ground or vegetation vs. avalanche that does not reveal the ground or vegetation, and avalanche that is blocked by vegetation or other objects having high roughness vs. avalanche that is not adjacent to any rough object. These topographic conditions influence the appearance of avalanches on images; therefore, the number of steps in our OBIA workflow is large. We found that OBIA is useful for complex shapes because it allows the implementation of assumptions regarding each different situation. Additionally, this OBIA algorithm may be used and modified according to the need of the user and the data. For example, some steps in our OBIA workflow may be omitted, such as those used for filling the gaps inside the avalanche. If the data do not contain avalanches that reveal bare ground or vegetation, these steps may not improve the classification because there will not be any gaps to reclassify. Additionally the number of loops in the shrinkage and growth steps depend on pixel size. With our $0.25 \mathrm{~m}$ resolution NIR images, we shrank and increased the segments of $1.25 \mathrm{~m}$, which was sufficient to split segments into small parts; with higher (lower) resolution, the number of loops should be increased (decreased) accordingly. Previous studies have reported very high accuracy in separating vegetation and water using vegetation and water indices (Townshend and Justice, 1986; Ji et al., 2009); therefore, we assume that the NDVI and NDWI thresholds are stable and may be easily transferable to other areas and data. Despite this, the thresholds for $\mathrm{SD}_{\mathrm{NDWI}}$ should always be verified by analysing the mode of data distribution on a histogram of the $\mathrm{SD}_{\mathrm{NDWI}}$, testing the thresholding of data samples, and checking the thresholding results visually. We are aware that such visual checking may introduce some bias, although so far it is the most common way in OBIA to find the most suitable threshold.

The size and the shape of avalanches did not influence the classification accuracy when they were bigger than 2000 pixels, which is what we regard as the minimum detectable size in our OBIA algorithm. We selected the minimum number of pixels necessary by analysing the reference data, where avalanches smaller than 2000 pixels constituted only $7.5 \%$ of the total number of avalanches, and by taking into account the classification of avalanches with respect to their size. According to Bühler et al. (2009), very small avalanches are $<100 \mathrm{~m}^{-2}$, which coincides with 2000 pixels $\left(125 \mathrm{~m}^{-2}\right)$. The parameters playing the biggest roles were brightness and the $\mathrm{SD}_{\mathrm{NDWI}}$ (Fig. 6). Visual inspection showed that the easiest avalanche part to detect was the run-out zone and the most difficult was the release zone, because release zones were not usually rough enough or did contain outcrops of the vegetation or bare ground. The correct detection of the track depended mostly on snow roughness and depth. In a case of low roughness values or a very thin snow cover revealing the ground and the vegetation, the track was not detected correctly or not detected at all.

Our method correctly identified $61 \%$ of the total avalanche area in the study area mainly because of heterogeneous avalanche debris with dark or smooth patches and also because of differing deposit ages and hence differing surface roughness. The most difficult to classify were old avalanches where the bare ground cropped out or where vegetation occurred in the path of the avalanche. These avalanches did not meet the assumptions in our OBIA protocol and could not be classified correctly because they were not rough enough or were too vegetated or dark due to thin snow cover. Here a solution may be using different thresholds for old and fresh avalanches to optimise our OBIA approach. We tested different thresholds for the input layers and different neighbourhood assumptions to include these avalanches; however, this resulted in more false positives, so the cost of correct classification of these avalanches was higher than the benefit. We therefore decided to stay with the same workflow and thresholding shown in Fig. 4 for the whole study area.

Errors in automatic classification also occurred due to data tiling. Using an Intel Xeon E5-2667U processor with 256 GB RAM memory, we were able to run our OBIA algorithm only for tiles of $6.25 \mathrm{~km}^{-2}$ (10000 columns $\times 10000$ rows), requiring a computing time of $\sim 30 \mathrm{~min}$ for each tile; our test in executing bigger tiles ended in crashing the computation in the eCognition software. In many cases the avalanches were therefore split into two or more neighbouring tiles, which influenced the correct detection of avalanche parts, especially those where the avalanches were spread across tiles or had small gaps.

Avalanches often have a tongue shape that may also be used as a property for classifying. In our test area, however, using such information may be insufficient, because we are dealing with avalanches repeated in the same location and merged avalanches, which have more complex shapes. In such cases, a new avalanche may partly cover previous avalanche, making it difficult to distinguish them. In addition, a few avalanches may simply have a more complex and difficult-to-interpret shape. Detailed enough maps of snow thickness may help to improve the classification accuracy, and perhaps enable separating release zones, tracks, and runout zones from the surrounding snow cover. Our estimated avalanche density map and information concerning the most common prevalence of avalanches may be used to help gen- 
erate a hazard map in mountainous areas. The most crucial issue, however, is whether the size or the number of avalanches is more important in such mapping. As mentioned by Eckerstorfer et al. (2016), only very small avalanches cause less damage; therefore, we suggest weighting the density map according to avalanche size to give more information regarding the degree of danger in a specific area.

Continuation of our research should contain verification of the transferability of our OBIA algorithm to data from different winter years for the area of Davos, Switzerland, which we have in our repository. Future tests involving diverse areas across the world and different types of data (e.g. UAV, radar, and seismic) are desirable. An approach for distinguishing single and repeat avalanches at the same area should be developed, because it would give more detail about the quantity and the frequency of avalanches in a given area.

\section{Conclusions}

We have presented an automatic OBIA approach to detecting snow avalanches and their release, track, and deposition zones for a large region from ADS80 NIR aerial images. We used image-derived parameters - the NDVI and the NDWI and its standard deviation $\left(\mathrm{SD}_{\mathrm{NDWI}}\right)$ - to separate vegetation, snow, and rough snow representing avalanche debris, respectively. We applied buffering assumptions relying on the local neighbourhood of segments to remove salt-and-pepper noise and objects that were falsely assigned as avalanches, using the thresholding of derivatives obtained from NIR images. For an area of $226.3 \mathrm{~km}^{-2}$, our algorithm achieved producer's and user's accuracies of 0.61 and 0.78 , respectively, and a Cohen's kappa of 0.67. Our algorithm uses only information taken from these images, from which the evaluated NDVI and NDWI indices are normalised, helping to transfer their thresholds to other areas. Our approach contains only three fixed parameters (NDVI, NDWI, and $\mathrm{SD}_{\mathrm{NDWI}}$ ) and two changeable parameters (brightness and segment area). The first depend on the spectral characteristics of images and the latter one depend on the resolution of images. Our OBIA workflow is not sequential when compared to that of Lato et al. (2012); the segments that do not fulfil assumptions in one step can still be considered as potential snow avalanches in the next steps. To assign potential snow avalanches, our method takes into account only the rough snow, which allows more reliable detection of avalanches. Our probability approach determines, in an automatic way, the highest and the lowest parts of the avalanche and thus its release zone, track, and run-out zone, which allows easy analysis of the topographic condition of areas where the avalanche starts and where the snow is deposited. For multiple avalanches with a complex shape, our probability map may not be sufficient to correctly identify all release and run-out zones, but for single avalanches it gives valuable results. For some avalanches, we were not able to judge visually whether they were single or multiple; a discussion of this topic should therefore be undertaken. The probability approach may be used for any other mass movement landforms, such as landslides, to delineate their release and deposition zones. In the future, we plan to validate our snow-avalanche algorithm for ADS data, which we have for other winters, and to verify its transferability to other NIR images, because successful results in this matter may offer a chance to improve hazard maps and avalanche forecasting in Switzerland.

Data availability. The data and the results of our work are available under the following link: https: //uni-potsdam.maps.arcgis.com/apps/Cascade/index.html?appid= 3b5ac4491b59480c8c6016139f285e88, Snow Avalanches, Karolina Korzeniowska, 2017.

Competing interests. The authors declare that they have no conflict of interest.

Acknowledgements. This research was funded by the European Union under the Marie Curie Initial Training Network ALErT (Creation of an interactive CAP natural-hazard database), project number FP7-PEOPLE-2013-ITN-607996. The ADS80-SH92 airborne images used in the study were provided by the WSL Institut für Schnee- und Lawinenforschung SLF, Davos, and Leica Geosystems AG (Rüdiger Wagner, Norbert Lämmer, Fernando Schapira). The authors would like to thank Julia Wessels and Benjamin Zweifel for providing historical snow-avalanche data for Switzerland and the two referees for their comments.

Edited by: Rosa Lasaponara

Reviewed by: Olivier Jaquet and one anonymous referee

\section{References}

Badoux, A., Andres, N., Techel, F., and Hegg, C.: Natural hazard fatalities in Switzerland from 1946 to 2015, Nat. Hazards Earth Syst. Sci., 16, 2747-2768, https://doi.org/10.5194/nhess16-2747-2016, 2016.

Bagli, S. and Schweizer, J.: Characteristics of wet-snow avalanche activity: 20 years of observations from a high alpine valley (Dischma, Switzerland), Nat. Hazards, 50, 97-108, https://doi.org/10.1007/s11069-008-9322-7, 2009.

Bründl, M., Etter, H.-J., Steiniger, M., Klingler, Ch., Rhyner, J., and Ammann, W. J.: IFKIS - a basis for managing avalanche risk in settlements and on roads in Switzerland, Nat. Hazards Earth Syst. Sci., 4, 257-262, https://doi.org/10.5194/nhess-4257-2004, 2004.

Bühler, Y., Hüni, A., Christen, M., Meister, R., and Kellerberger, T.: Automated detection and mapping of avalanche deposits using airborne optical remote sensing data, Cold Reg. Sci. Technol., 57, 99-106, https://doi.org/10.1016/j.coldregions.2009.02.007, 2009. 
Bühler, Y., Meier, L., and Ginzler, C.: Potential of operational high spatial resolution near-infrared remote sensing instruments for snow surface type mapping, IEEE Geosci. Remote Sens. Lett., 12, 821-825, https://doi.org/10.1109/LGRS.2014.2363237, 2015.

Bühler, Y., Adams, M. S., Bösch, R., and Stoffel, A.: Mapping snow depth in alpine terrain with unmanned aerial systems (UASs): Potential and limitations, Cryosphere, 10, 1075-1088, https://doi.org/10.5194/tc-10-1075-2016, 2016.

Bühler, Y., Adams, M. S., Stoffel, A., and Boesch, R.: Photogrammetric reconstruction of homogenous snow surfaces in alpine terrain applying near-infrared UAS imagery, Int. J. Remote Sens., 0, 1-24, https://doi.org/10.1080/01431161.2016.1275060, 2017.

Castebrunet, H., Eckert, N., and Giraud, G.: Snow and weather climatic control on snow avalanche occurrence fluctuations over $50 \mathrm{yr}$ in the French Alps, Clim. Past, 8, 855-875, https://doi.org/10.5194/cp-8-855-2012, 2012.

Christen, M., Kowalski, J., and Bartelt, P.: RAMMS: Numerical simulation of dense snow avalanches in threedimensional terrain, Cold Reg. Sci. Technol., 63, 1-14, https://doi.org/10.1016/j.coldregions.2010.04.005, 2010.

Cohen, J.: A coefficient of agreement for nominal scales, Educ. Psychol. Meas., 20, 37-46, 1960.

Congalton, R. G.: A review of assessing the accuracy of classifications of remotely sensed data, Remote Sens. Environ., 37, 35-46, https://doi.org/10.1016/0034-4257(91)90048-B, 1991.

Eckerstorfer, M. and Malnes, E.: Manual detection of snow avalanche debris using high-resolution Radarsat-2 SAR images Radarsat-2 SAR images, Cold Reg. Sci. Technol., 120, 205-218, https://doi.org/10.1016/j.coldregions.2015.08.016, 2015.

Eckerstorfer, M., Bühler, Y., Frauenfelder, R., and Malnes, E.: Remote sensing of snow avalanches: Recent advances, potential, and limitations, Cold Reg. Sci. Technol., 121, 126-140, https://doi.org/10.1016/j.coldregions.2015.11.001, 2016.

Ji, L., Zhang, L., and Wylie, B.: Analysis of dynamic thresholds for the normalized difference water index, Photogramm. Eng. Remote Sens., 75, 1307-1317, https://doi.org/10.14358/PERS.75.11.1307, 2009.

Larsen, S. Ø., Salberg, A., and Solberg, R.: Automatic avalanche mapping using texture classification of optical satellite imagery, edited by: Lasaponara, R., Masini, N., and Biscione, M., EARSeL, 2013, Towards Horizon 2020, 399-410, 2013.

Lato, M. J., Frauenfelder, R., and Bühler, Y.: Automated detection of snow avalanche deposits: segmentation and classification of optical remote sensing imagery, Nat. Hazards Earth Syst. Sci., 12, 2893-2906, https://doi.org/10.5194/nhess12-2893-2012, 2012.
McClung, D. and Schaerer, P.: The Avalanche Handbook, The Mountaineers Book, Seattle, 2006.

McClung, D. M.: Avalanche character and fatalities in the high mountains of Asia, Ann. Glaciol., 57, 114-118, https://doi.org/10.3189/2016AoG71A075, 2016.

McFeeters, S. K.: The use of the normalized difference water index (NDWI) in the delineation of open water features, Int. J. Remote Sens., 17, 1425-1432, https://doi.org/10.1080/01431169608948714, 1996.

Schweizer, J. and Jamieson, J. B.: Snow cover properties for skier triggering of avalanches, Cold Reg. Sci. Technol., 33, 207-221, https://doi.org/10.1016/S0165-232X(01)00039-8, 2001.

Schweizer, J., Jamieson, J. B., and Schneebeli, M.: Snow avalanche formation, Rev. Geophys., 41, 1-25, https://doi.org/10.1029/2002RG000123, 2003.

Sithole, G. and Vosselman, G.: Experimental comparison of filter algorithms for bare-Earth extraction from airborne laser scanning point clouds, ISPRS J. Photogramm., 59, 85-101, https://doi.org/10.1016/j.isprsjprs.2004.05.004, 2004.

Techel, F., Zweifel, B., and Winkler, K.: Analysis of avalanche risk factors in backcountry terrain based on usage frequency and accident data in Switzerland, Nat. Hazards Earth Syst. Sci., 15, 19851997, https://doi.org/10.5194/nhess-15-1985-2015, 2015.

Techel, F., Jarry, F., Kronthaler, G., Mitterer, S., Nairz, P., Pavsek, M., Valt, M., and Darms, G.: Avalanche fatalities in the European Alps: Long-term trends and statistics, Geogr. Helv., 71, 147-159, https://doi.org/10.5194/gh-71-147-2016, 2016.

Townshend, J. R. G. and Justice, C. O.: Analysis of the dynamics of African vegetation using the normalized difference vegetation index, Int. J. Remote Sens., 7, 1435-1445, https://doi.org/10.1080/01431168608948946, 1986.

Varma, M. and Zisserman, A.: A statistical approach to texture classification from single images, Int. J. Comput. Vision, 62, 61-81, https://doi.org/10.1007/s11263-005-4635-4, 2004.

Vickers, H., Eckerstorfer, M., Malnes, E., Larsen, Y., and Hindberg, H.: A method for automated snow avalanche debris detection through use of Synthetic Aperture Radar (SAR) imaging, Earth Space Sci., 3, 446-462, https://doi.org/10.1002/2016EA000168, 2016.

Wilhelm, C., Wiesinger, T., Bründl, M., and Ammann, W.: The avalanche winter 1999 in Switzerland - An overview, Proc. of the 2000 International Snow Science Workshop, Montana, USA, 487-494, 1999. 